Supporting Information

for

\title{
Self-organized Interconnect Method for Molecular Devices
}

by

\begin{abstract}
Masateru Taniguchi, ${ }^{\dagger}$ Yoshihiro Nojima, ${ }^{\dagger}$ Kazumichi Yokota, ${ }^{\dagger}$
Jun Terao, ${ }^{\ddagger}$ Kimihiko Sato, ${ }^{\ddagger}$ Nobuaki Kambe, ${ }^{\ddagger}$ Tomoji Kawai ${ }^{\dagger}$
\end{abstract}

${ }^{\dagger}$ The Institute of Scientific and Industrial Research, Osaka University, 8-1 Mihogaoka, Ibaraki, Osaka 567-0047, Japan

taniguti@ sanken.osaka-u.ac.jp

kawai@sanken.osaka-u.ac.jp

ॠDepartment of Molecular Chemistry \& Science and Technology Center for Atoms, Molecules and Ions Control, Graduate School of Engineering, Osaka University, Yamadaoka 2-1, Suita, Osaka 565-0871, Japan

kambe@chem.eng.osaka-u.ac.jp 


\section{$\underline{\text { Synthesis of orientation control molecule (2) }}$}

A two-necked flask was charged with diiodide $(0.10 \mathrm{mmol})$, diboronic ester $(0.124 \mathrm{mmol})$, $\alpha$-cyclodextrin $(929 \mathrm{mg}, 0.818 \mathrm{mmol}), \mathrm{Pd}(\mathrm{OAc})_{2}(0.01 \mathrm{mmol})$, and $\mathrm{Li}_{2} \mathrm{CO}_{3}(0.6 \mathrm{mmol})$ and degassed water $(15 \mathrm{~mL})$ was added by syringe. The mixture was stirred over night at $85^{\circ} \mathrm{C}$ under nitrogen. The solution was diluted with water $(15 \mathrm{~mL})$ and passed through a $0.22 \mathrm{~mm}$ filter (cellulose ester membrane). The product was further purified from low-molecular weight material by ultrafiltration (polysulfone membrane; NMWCO $5 \mathrm{kDa}$ ), then evaporated to yield orientation controal molecule 2.

${ }^{1} \mathrm{H}-\mathrm{NMR}\left(400 \mathrm{MHz}, \mathrm{D}_{2} \mathrm{O}\right) \delta=8.5-7.0 \quad$ (aromatic-H, 136H), 4.2-3.2(CD-H, 238H).

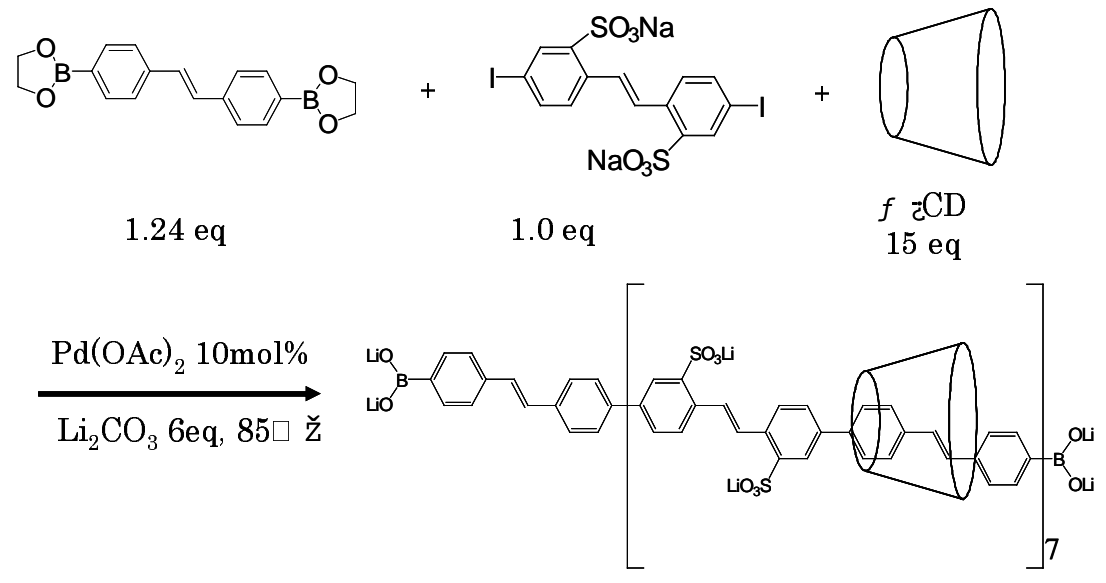

Scheme $\mathbf{S 1}$

\section{$\underline{\text { Experimental procedure of self-organized interconnect method }}$}


We placed the electrode substrate and nano-scale electrodes for $12 \mathrm{~h}$ in a methanol solution in which the interface control molecules 1 were dissolved, and then washed them with methanol. We placed the electrodes modified with the interface control molecules $\mathbf{1}$ in the solution of the orientation control molecules 2 , and agitated the solution all night at $85^{\circ} \mathrm{C}$. After the reaction, we removed the electrodes from the solution and washed them with ultrapure water. We placed $0.1 \mathrm{mmol}$ of the function control molecules 3 or 4 and $0.02 \mathrm{mmol}$ of $\mathrm{Pd}\left(\mathrm{PPh}_{3}\right)_{4}$ in a reaction vessel, whose inner gas was replaced by nitrogen gas. We then added a $2 \mathrm{M}$ potassium carbonate solution $(2 \mathrm{ml})$ and THF $(2 \mathrm{ml})$ and placed the electrodes in the solution. We agitated the solution all night at $50{ }^{\circ} \mathrm{C}$. After the reaction, we removed the electrodes from the solution and washed them with diethylether and ultrapure water.

After Step 2, we observed the molecular height in the perpendicular direction from the electrode surface and found that the mean molecular height was 13-14 $\mathrm{nm}$. The length of Molecule 3 and 4 was estimated as $1.4 \mathrm{~nm}$ by density functional theory(DFT) calculation. The perpendicular length from the electrode surface was therefore 14 x $2+1.4=29.4 \mathrm{~nm}$, i.e. approximately $30 \mathrm{~nm}$. As a control experiment, we conducted molecular wiring with the inter-electrode distance of $25 \mathrm{~nm}, 30 \mathrm{~nm}, 35 \mathrm{~nm}$, and $40 \mathrm{~nm}$, and found the electric conduction and optical switching function only when the distance was $30 \mathrm{~nm}$.

\section{$\underline{\text { Reproducibility }}$}

In our interconnect method we achieved reproducibility of about $30 \%$ when we set the criterion that 
electric conduction should be observed in the conductive wire and optical switching function should be found in the optical switching device.

\section{$\underline{\text { Electrical characterization }}$}

We measured electric conduction at room temperature with no light in a vacuum condition below $10^{-5}$ Torr. The electric noise level of the measurement was less than $10 \mathrm{pA}$. We placed the optical switching device in ultraviolet light or visible light and shielded them from light to measure the current-voltage characteristic of the device. We applied ultraviolet radiation to the molecules to drive them into the closed-ring state, and then applied visible light to switch them into the ring-opening state. The intensity of the ultraviolet light was $30 \mu \mathrm{W}$ at the wavelength $313 \mathrm{~nm}$, while that of the visible light was $30 \mu \mathrm{W}$ at $532 \mathrm{~nm}$.

\section{$\underline{\text { Nanogap electrodes }}$}

We used an electron beam lithography technique and the lift-off process to create the nano-scale electrodes from a silicon substrate with a $300 \mathrm{~nm}$ oxide silicon layer and gold/titanium electrodes.

The electrode metal was evaporated with the RF-magnetron sputtering method. The nano-scale electrodes were all cleaned in the UV-ozone. The inter-electrode spacing was $30 \mathrm{~nm}$.
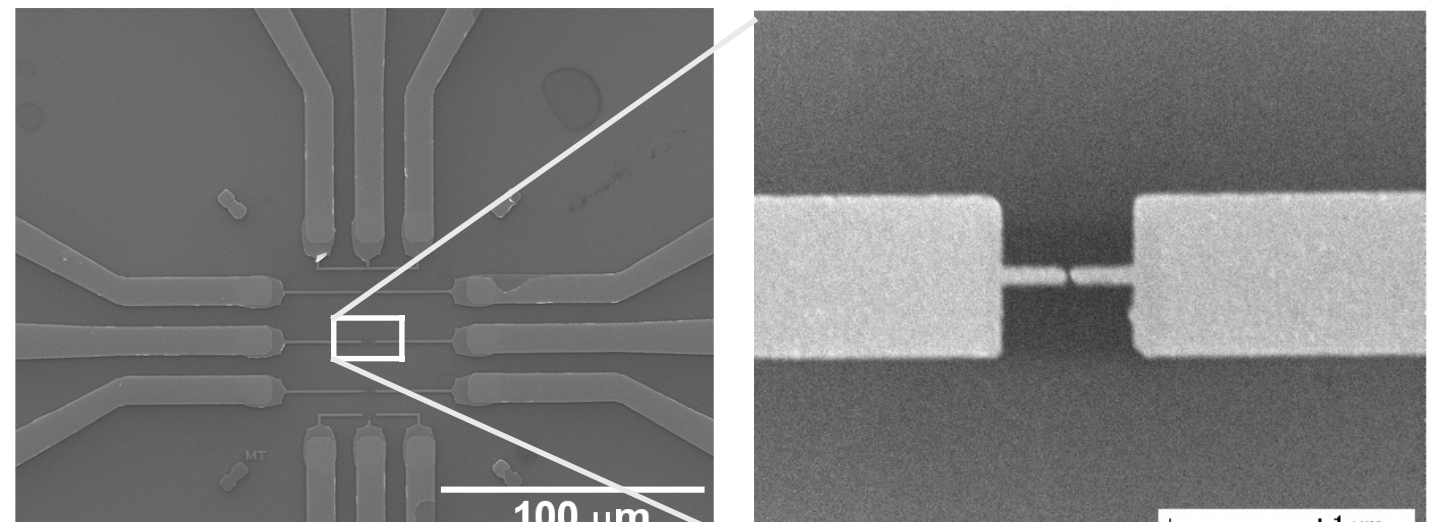
Figure S1. SEM images of nanogap electrodes, whose spacing was $30 \mathrm{~nm}$. 


\section{UV-Vis spectrum of the interface control molecules plus the orientation control molecules on}

\section{the gold substrate}

We measured the UV-Vis spectrum of the orientation control molecules in a solution, and that of the interface control molecules plus the orientation control molecules on the gold electrodes. The blue line represents the spectrum of the orientation control molecules and the red line shows that of the interface control molecules plus the orientation control molecules on the gold substrate. The measurement of the gold substrate was conducted in the reflection mode. The peak absorption of the orientation control molecules in the solution was found at wavelength $397 \mathrm{~nm}$, while that of the interface control molecules plus the orientation control molecules on the gold substrate was observed at $400 \mathrm{~nm}$. Due to the surface plasmons of the gold, the absorption curve in the measurement of the gold substrate was somewhat broadened around 500nm.

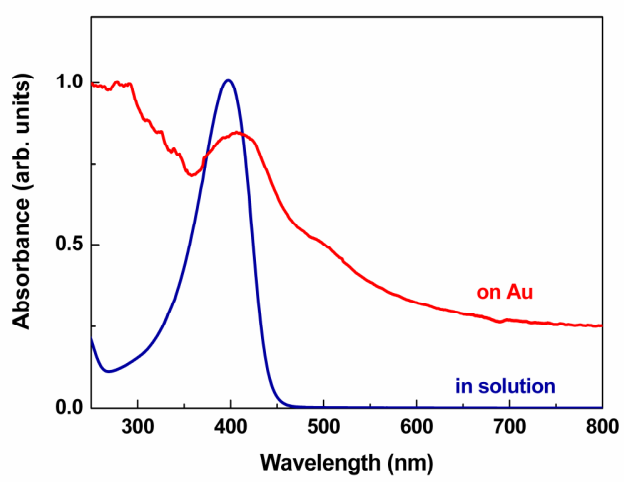

Figure S2. UV-Vis spectrum of the orientation control molecules in a solution, and that of the interface control molecules plus the orientation control molecules on the gold electrodes. 
(a)

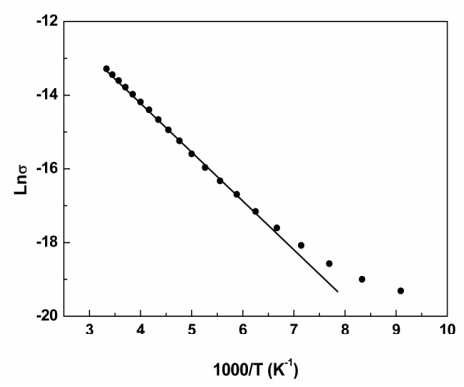

(b)

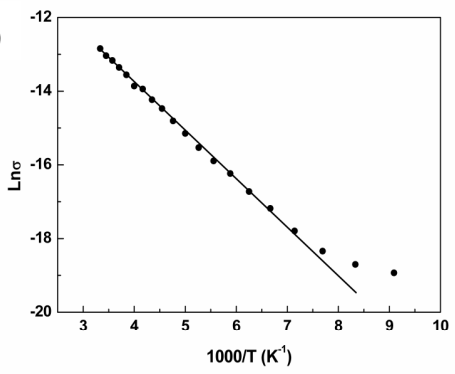

Figure S3. Temperature dependence of conductance of molecular wires. (a) the conductive wire and

(b) the optical switching device for which we used $\mathbf{3}$ and $\mathbf{4}$ as the functional molecule, respectively.

The electric conductivity showed Arrhenius type behavior in the high temperature range. The straight

line in the figure is the range that we used to calculate the activation energy.

\section{Time dependency of electric property of optical switching molecules}

(a)

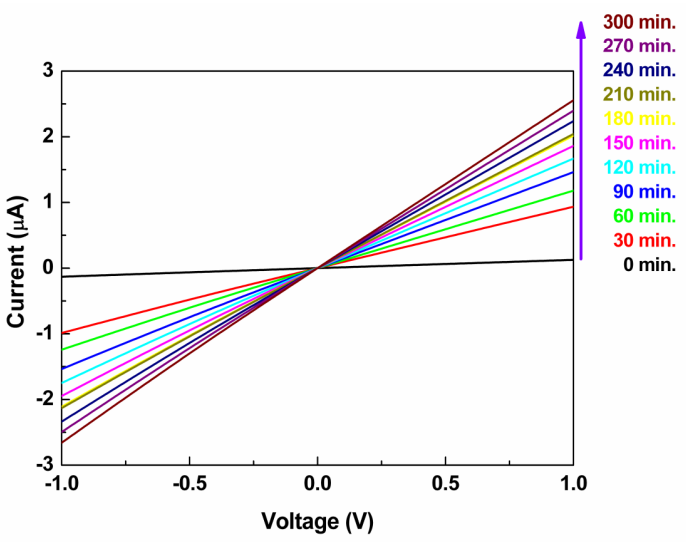


(b)

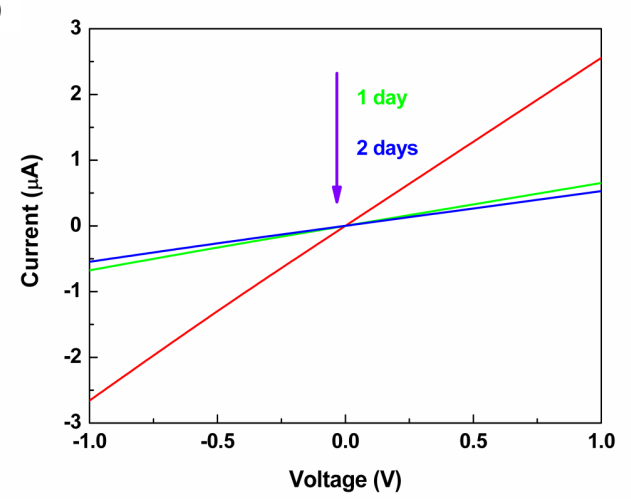

Figure S4. Time dependence of electric property of optical switching molecules under (a) the UV light and (b) the visible light.

After radiating light to the optical switching devices, we conducted electric measurements in a vacuum with no light. As shown in Figure S4(a), the UV radiation to the optical switching device wired between the nano-scale electrodes enhanced the electric current as the radiation time passed, and the current became saturated after about 300 minutes. In the visible light radiation the electric current did not decrease drastically in several minutes but decreased down to $25 \%$ of the amplified current in one day (Figure S4(b)). After turning ON the optical switching device under the UV light, we kept them for more than 24 hours in a vacuum with no light, measured the electric current, and did not find change the current. This suggests that the current's ON-OFF switching was not driven by the heat but by the light. The speed of the light-driven switching in a single molecule is usually expected to be fast, although, as reported in Ref. 13 and 14, the switching speed of our optical switching molecules was slow. There could be two reasons for the slow response speed of the switching molecules wired between the nano-scale electrodes. One is that in our experiment, the switching 
molecules were fixed between electrodes and hence the molecular devices were structurally restricted.

So the ring opening/closing structural change of the optical switch was also restricted, causing the slow response speed. The second reason is that the $\pi$-electrons of our molecular wires were covered with $\alpha$-cyclodextrin and the $\pi$-electrons were spatially distant. Energy transfer between the molecules plays an important role in the ring opening/closing reactions ${ }^{*}$. Therefore the energy transfer between the molecules was suppressed in the molecular wires with the optical switching function and accordingly the response speed was slow.

\section{References}

(*)Tsujioka, T.; Kume, M.; Irie, M. J. Photochem. Photobiol. A: Chem. 1997, 104, 203-206. 